\title{
Sentries at the gate: chemokines and the blood-brain barrier
}

\author{
Andrzej R Glabinski ${ }^{1}$ and Richard M Ransohoff*,2 \\ ${ }^{1}$ Department of Neurology, Medical University of Lodz, Lodz, Poland; ${ }^{2}$ Department of Neurosciences, The Lerner \\ Research Institute, Cleveland Clinic Foundation, 9500 Euclid Avenue, Cleveland, OH 44195, USA
}

Keywords: chemotactic factors; inflammation; wounds and injuries; BBB

\section{The blood-brain barrier and immune cell homing to the CNS}

The blood-brain barrier (BBB) isolates the CNS from the rest of the organism. The anatomic foundation of this structure is a syncytium of cerebrovascular endothelial cells sealed together by tight junctions. The endothelial basal lamina, located at the abluminal side of endothelium, borders the perivascular (Virchow-Robin) space. The perivascular space is continuous with the subarachnoid compartment and is itself bordered by the glia limitans, formed by astrocytic and microglial end feet. Within the perivascular space, on either side of the endothelial basal lamina, dwell a unique population of phagocytic cells variously termed perivascular macrophages, perivascular microglia, perivascular cells or pericytes. These cells form a first line of defense once the $\mathrm{BBB}$ is breached. Astrocytes are also important for BBB development and function: their processes attach to the basement membrane shared with the endothelial cells and perivascular cells. The close contact between astrocytes and brain endothelial cells suggests that both cell types interact in maintaining the function of the BBB, a bidirectional interaction that is mediated at least in part by cytokine-mediated signaling (Selmaj, 1996; Johansson, 1990).

It is known that activated lymphocytes cross the intact BBB in the process of executing immunological surveillance of the CNS. T-cell migration into the normal CNS is antigen nonspecific and is contingent solely upon the activation state of the lymphocyte (Hickey et al, 1991; Wekerle et al,

${ }^{*}$ Correspondence: RM Ransohoff

Received 4 August 1999; revised 8 September 1999; accepted 23 September 1999
1986). Leukocyte transmigration through a vascular endothelium involves a well-characterized multistep process of cell-cell interactions. Initial interactions between selectins on leukocytes and their receptors on endothelial cells slows down the circulating hematogenous cells, a state termed 'rolling'. Rolling is permissive for activation of adhesion molecules (leukointegrins), which promote tight adhesion of leukocytes to vascular endothelium. Finally inflammatory cells migrate through endothelium along concentrations gradients of chemokines (Springer, 1994).

Lymphocytes bind to CNS endothelial cells with lower affinity than to endothelium derived from other organs. After stimulation with proinflammatory cytokines lymphocyte adhesion to cerebrovascular endothelium increases and becomes similar to that observed in other organs (Male et al, 1990). Perhaps, this fact may explain why during CNS inflammatory conditions the $\mathrm{BBB}$ endothelium becomes permeable for hematogenous inflammatory cells.

Expression of the VLA-4 integrin by CD4 T cells is required for their migration into brain parenchyma in EAE. Moreover, antibodies to VLA-4 and its ligand VCAM-1 ameliorated pathogenicity of encephalitogenic cells in vivo (Baron et al, 1993; Yednock et al, 1992). A great deal of investigation of the BBB in inflammation has focused on EAE. After BBB penetration and local encounter with antigens, lymphocytes initiate inflammatory processes within the CNS parenchyma. It has been shown that there are two stages in homing of $\mathrm{T}$ lymphocytes to the CNS during EAE: initial selective and second nonselective, when clinical signs appear (Karin et al, 1993). Only a small number of inflammatory 
cells accumulating in the CNS at the onset of EAE are antigen-specific. Studies with radioactively labeled MBP-specific $\mathrm{T}$ cells showed that they represent only 1 to $4 \%$ of the inflammatory cell population in the CNS at the onset of EAE. Furthermore, labeled cells remained primarily within the perivascular compartment (Cross et al, 1990). The pathogenic role for antigen specific $T$ cells may thus be exerted by promoting migration of antigen-nonspecific inflammatory cells from the blood to the CNS parenchyma.

\section{Chemokines and their receptors - a brief overview}

Chemokines are small $(8-12 \mathrm{kD})$ chemoattractant cytokines which can stimulate directional migration of cells. Traditionally, the chemokine family can be divided into four separate subfamilies according to the position of the first two cysteines near the $\mathrm{N}$ terminus. In addition to differences in structure, there are also functional differences between subfamilies. In CXC or alpha- chemokines, the first two cysteines are separated by one additional amino acid. In humans CXC chemokine genes are clustered on chromosome 4 . They can be further divided into two groups; those with ELR (glutamateleucine-arginine) motif preceding the first cysteine are primarily chemoattractant for neutrophils, while non-ELR alpha-chemokines attract activated lymphocytes and varied other cells. CC or betachemokines have the first two conserved cysteines adjacent to one another. Their genes are mainly clustered on chromosome 17 in humans and they attract principally mononuclear inflammatory cells. Recently two new chemokine subfamilies with one member each were described. The $\mathrm{C}$ or gammachemokine subfamily consists of lymphotactin, which is a potent $\mathrm{T}$ lymphocyte chemoattractant. Its gene maps to chromosome 1 in human genome. The single member of the CX3C or delta- chemokine subfamily, named fractalkine, has a unique structure because of the presence of three amino acids intervening between the first two cysteines. Fractalkine, unlike other chemokines, is a membrane bound protein with a chemokine domain at its N-terminus, which can be cleaved and may chemoattract mononuclear leukocytes (Luster, 1998; Rollins, 1997).

All chemokine receptors belong to the seven transmembrane spanning, G-protein coupled receptor family. So far, five CXC and nine CC chemokine receptors have been cloned (Rollins, 1997). Additionally, one receptor each for fractalkine and lymphotactin have been identified. Most of the chemokine receptors display overlapping specificities. Several CC and two out of five CXC chemokine receptors (CXCR2 and CXCR3) can bind more than one ligand. The only ligand specific chemokine receptors are CXCR1 (IL-8 receptor), CXCR4 (SDF-1 receptor) and CXXXCR1 (fractalkine receptor). With few exceptions, the signaling chemokine receptors are family specific. A major anomaly is a non-signaling receptor, termed DARC (Duffy Antigen Receptor for Chemokines). This receptor is localized on erythrocytes and postcapillary venules and binds both CXC and CC chemokines. As DARC does not signal upon binding ligands, its physiological function remains obscure. Several DNA viruses encode molecules homologous to chemokine receptors. Well known examples are Herpesvirus saimiri ECRF3 (encoding a protein similar to CXCR1 and CXCR2) and Cytomegalovirus. US28 encoding a polyvalent CCR1-like molecule. It has been suggested that mimicking chemokine receptors may be advantageous for viruses to inactivate self-defense mechanisms of the infected host (Ahuja et al, 1994; Ahuja and Murphy, 1993; Luster, 1998; Rollins, 1997).

\section{Chemokines and nervous-system inflammation}

Chemokine expression within CNS and PNS tissues is commonly observed in the context of inflammation. In the following subsections, we summarize several studies of chemokine expression and function in model and clinical neurological disorders. This information provides a foundation for considering how chemokine expression affects the function of the BBB.

\section{Chemokines in autoimmune CNS inflammation}

Any process damaging the BBB enables blood cellular and molecular components to gain access to the CNS and initiate inflammatory responses. This process occurs in mechanical, vascular and infectious injury to the CNS. Much more obscure is the mechanism leading to CNS inflammation without initial disruption of the BBB. This situation is observed at the early stages of autoimmune inflammation in multiple sclerosis (MS) and its animal model experimental autoimmune encephalomyelitis (EAE).

It has been proposed that the prime triggering factor for the initiation of autoimmune CNS inflammation consists of antigen-specific $\mathrm{T}$ cells. After BBB penetration they may stimulate astrocytres and other CNS cells to produce chemokines (Tani, 1994; Glabinski et al, 1995a). Additionally, encephalitogenic $\mathrm{T}$ cells produce abundant levels of MCP-1, MIP- $1 \alpha$ and RANTES (Godiska et al, 1995; Kuchroo et al, 1993; Sun et al, 1997). This chemokine gradient favors migration of other mononuclear leukocytes from the circulation to the CNS compartment. Chemokine expression was detected in EAE only when inflammatory cells were already present in the CNS (Glabinski et al, 1995b). The influx of a large population of inflammatory 
cells is necessary for disease manifestation as shown in animals with depletion of effector cells from the circulation (Brosnan et al, 1981).

Six years ago, the first studies described increased expression of some chemokines at the onset of EAE (Hulkower et al, 1993; Ransohoff et al, 1993). Chemokines MCP-1 and IP-10 were highly expressed in mouse and rat models of EAE. In the mouse model, astrocytes were the cellular source of chemokine expression as showed by in situ hybridization/immunohistochemistry colocalization studies (Ransohoff et al, 1993; Tani et al, 1996a,b). Later, additional chemokines were also detected in the CNS of animals shortly before clinical signs of EAE (Godiska et al, 1995) and expression of some chemokines correlated with the intensity of CNS inflammation (Glabinski et al, 1998). Our studies suggested that chemokines produced by astrocytes were responsible for amplification of inflammatory cell invasion of the CNS but not its initiation (Glabinski et al, 1995b). In chronic relapsing EAE (ChREAE) we observed increased expression of several chemokines during spontaneous relapse of the disease as compared with disease remission. Some chemokines (MCP-1, IP-10 and GRO- $\alpha$ ) were expressed by astrocytes, other (RANTES, MIP-1 $\alpha$ ) by inflammatory cells (Glabinski et al, 1997). Fractalkine and its receptor were also upregulated in EAE (Jiang et al, 1998; Pan et al, 1997). Expression of some chemokines by CNS parenchymal cells in the EAE model is probably stimulated by proinflammatory cytokines as shown by studies in mice with interferon gamma knock-out and EAE. In that model IP-10 expression was selectively diminished in contrast with two other chemokines, MCP-1 and GRO- $\alpha$ (Glabinski et al, 1999). The importance of chemokines in EAE pathogenesis was confirmed by experiments showing disease amelioration after treatment with antichemokine antibodies (Karpus and Ransohoff, 1998; Kennedy et al, 1998; Karpus and Kennedy, 1997; Karpus et al, 1995). In these studies, anti-MIP$1 \alpha$ antibody inhibited development of acute EAE, whereas anti-MCP-1 antibody reduced the severity of relapsing EAE. An important complementary study showed increased expression of chemokine receptors CCR2, CCR5, CXCR4, CXXXCR1 in the spinal cords of animals with EAE (Jiang et al, 1998).

Recent reports addressed expression of chemokines within the CNS during MS. MIP- $1 \alpha$ concentrations in the CSF were statistically different from control during MS relapse and other inflammatory neurological diseases but in all cases was present at sub-picomolar levels (Miyagishi et al, 1995). MCP-1 was found in inflammatory cells and astrocytes in active plaques and astrocytes in surrounding white matter. Moreover, MIP- $1 \alpha$ and MIP- $1 \beta$ were present in inflammatory cells in active MS plaques (Simpson et al, 1998). MCP-1, MCP-2 and MCP-3 were also detected in reactive astrocytes and inflamma- tory cells in active acute and chronic MS lesions (McManus et al, 1998a). RANTES was localized by ISH to perivascular inflammatory cells (Hvas et al, 1997).

We found increased expression of chemokines IP10, Mig and RANTES in cerebrospinal fluid from patients with MS relapse (Sørensen et al, 1999). Additionally, we localized cells bearing receptors for those chemokines within MS plaques. CXCR3 (IP-10/Mig receptor) was present on lymphocytes within perivascular inflammatory cuffs; CCR5 (a RANTES receptor) was present on lymphocytes, macrophages and microglial cells in active MS lesions (Sørensen et al, 1999). Compatible results were reported by Balashov et al (1999), who additionally demonstrated increased production of IFN- $\gamma$ by circulating CCR $5+\mathrm{T}$ cells.

The implications of studying chemokines and their receptors in CNS immune-mediated inflammation are only provisional at best. The tight relationship between disease activity and chemokine expression in EAE is impressive, as is the correspondence between populations of infiltrating cells and production of appropriate chemokines. Abrogation or modulation of disease by antichemokine antibodies also supports the role of these cytokines in EAE pathogenesis. The recent results obtained from studying MS imply the possibility of using chemokine receptor blockade to treat this serious and often disabling disorder. However, the specific roles of chemokines and their receptors in this complex pathology (chemoattraction; activation; mitogenesis) remain to be defined.

Chemokines in physical and chemical CNS injury Chemokines are expressed in the CNS in diverse models of physical insult. Astrocytes were the main producers of MCP-1 shortly after penetrating mechanical injury to the brain (Berman et al, 1996; Glabinski et al, 1996). This expression was detected at the level of message as well as protein (Berman et al, 1996; Glabinski et al, 1998) and was localized on astrocytes in the vicinity of the lesion within hours after trauma (Glabinski et al, 1996). Other chemokines were not expressed at detectable levels in that injury model. Astrocytic expression of MCP-1 preceded influx of inflammatory cells to sites of injury and was present only in models with subsequent inflammatory reaction; thus, we observed only minimal expression of MCP-1 in a neonatal stab injury model characterized by lack of inflammatory cell migration to the injury site (Glabinski et al, 1996). In other studies, RANTES and MIP-1 $\beta$ were detected 1 day after stab injury to the brain, using immunohistochemistry which localized MIP-1 $\beta$ in reactive astrocytes and macrophages at the site of injury, while RANTES was diffusely expressed in necrotic tissue (Ghirnikar et $a l, 1996)$. When cortical lesion was augmented with lipopolysaccharide (LPS), many chemokines (MIP- 
$1 \alpha$, MIP-1 $\beta$, RANTES, IP-10, KC) were expressed in addition to MCP-1 (Hausmann et al, 1998). Neutralization of MCP-1 activity after stab wound injury to the brain with antisense oligodeoxynucleotides reduced by $30 \%$ the number of accumulated macrophages, demonstrating the potential of therapeutic modulation of posttraumatic inflammation in the brain using anti-chemokine strategies (Ghirnikar et al, 1998). Myelin edema chemically induced by triethyltin (TET) was shown to be associated with very rapid overexpression of proinflammatory cytokines including MIP-1 $\alpha$ (Mehta et al, 1998).

In experimental spinal cord injury MIP- $1 \alpha$ and $\beta$ were upregulated biphasically. In the early phase, $24 \mathrm{~h}$ after injury, chemokine expression was diffuse within the spinal cord gray matter. Later (4 days after injury), chemokine expression was restricted to inflammatory cells in proximity to the lesion (Bartholdi and Schwab, 1997). Cryogenic injury to the brain also increased expression of MCP-1 in the injured hemisphere, with a peak at $6 \mathrm{~h}$ post-injury and return to baseline by $48 \mathrm{~h}$ (Grzybicki et al, 1998). In the unlesioned hemisphere MCP-1 was also expressed, albeit to a lesser extent. We did not observe expression of IP-10 in this injury model (Grzybicki et al, 1998).

In a clinical study, CSF IL-8 concentrations were significantly higher in patients with severe traumatic brain injury than in corresponding sera, suggesting intrathecal production of this chemokine. CSF IL-8 levels correlated with BBB dysfunction measured by $\mathrm{CSF} /$ serum albumin ratio and nerve growth factor (NGF) production, suggesting an important role for IL-8 in the pathogenesis of brain injury (Kossmann et al, 1997).

It is notable that chemokines are rapidly, selectively and abundantly made in traumatized neural tissue, by resident cells such as astrocytes and Schwann cells. The study of chemokines in posttraumatic inflammation holds significant promise for devising methods to ameliorate the burden of trauma-associated disability. It will probably be possible to devise models of trauma that lack production of chemokines (in chemokine-deficient, gene-targeted mice) or response to chemokines (in receptor knockouts). These models will help to determine in which situations chemokine reduction or chemokine receptor blockade will be beneficial. In some select circumstances, the production of chemokines may be beneficial, and could be therapeutically augmented or substituted by chemokine mimicks.

\section{Chemokines in vascular CNS injury}

Increased expression of chemokines in the CNS was observed in varied experimental models of cerebral ischemia. Locally expressed in ischemic brain tissue, chemokines may be responsible for attraction of inflammatory cells and may contribute to tissue injury. In an early study, increased mRNA expression for MCP-1 and MIP-1 $\alpha$ was detected as early as $6 \mathrm{~h}$ after onset of cerebral ischemia (Kim et al, 1995). MCP-1-positive cells had the morphology of endothelial cells and macrophages, while MIP$1 \alpha$-positive cells resembled astrocytes (Kim et al, 1995). Others also observed increased MCP-1 expression in the ischemic cortex beginning $6 \mathrm{~h}$ after middle cerebral artery occlusion (MCAO) (Wang et al, 1995). From 6 h to 2 days after MCAO astrocytes surrounding the ischemic tissue were the main cellular source of MCP-1; after 4 days MCP-1 was detected in macrophages and microglia in the ischemic area (Gourmala et al, 1997). MIP-1 $\alpha$ expression was increased $4-6 \mathrm{~h}$ after experimental brain ischemia and localized in microglial cells within the ischemic region (Takami et al, 1997). Expression of the rat GAO- $\alpha$ homolog CINC (cytokine-induced neutrophil chemoattractant) was increased in the ischemic cerebral cortex 6$12 \mathrm{~h}$ after MCAO. No significant expression of CINC was detected in contralateral cortex (Liu et al, 1993). Interestingly, serum level of CINC was also transiently elevated 3-6 h after brain reperfusion injury (Yamasaki et al, 1995). In neonatal model of hypoxic-ischemic injury to the brain MCP-1 was first detected in periventricular regions of the lesioned hemisphere $1 \mathrm{~h}$ after ischemia. Peak of mRNA expression was observed at $8-24 \mathrm{~h}$, and completely resolved by 48 h (Ivacko et al, 1997).

Reperfusion after transient focal brain ischemia induces accumulation of neutrophils and severe brain edema. Depletion of neutrophils diminished the size of damaged area showing that neutrophils are primary mediators of reperfusion injury (Romson et al, 1983). Expression of neutrophil chemotactic cytokines IL-8 and CINC was shown to be increased after reperfusion (Matsumoto et al, 1997; Yamasaki et al, 1995). Administration of anti-IL-8 antibody significantly reduced brain edema and the volume of infarcted tissue (Matsumoto et al, 1997). Increased MCP-1 expression was also reported in rat forebrain reperfusion model, and was detected by RT/PCR as early as $1 \mathrm{~h}$ after reperfusion (Yoshimoto et al, 1997). Clinical studies showed that MCP-1 and IL-8 levels were evaluated in the CSF of patients operated for intracranial aneurysms. In patients with unruptured aneurysms, chemokine level was very low but increased significantly after subarachnoid haemorrhage (Gaetani et al, 1998).

In common with other determinants of cellular infiltration and activation (such as adhesion molecules), chemokines are considered potential therapeutic targets in cerebral ischemia. No 'proof-ofprinciple' experiments have been reported, however.

\section{Chemokines in CNS infections}

Clearly chemokine production by CNS and PNS cells has the evolutionary advantage of providing 
rapid and selective accumulation of leukocytes to a site of infection. Thus, the role of chemokines in host defense against pathogens has been characterized in multiple models of neural and extraneural infection. Not surprisingly, much of this effort has been devoted to understanding the beneficial and pathogenic roles of chemokines in the setting of chronic infection with HIV and related viruses.

Viral infection of the CNS parenchyma strongly stimulates local chemokine expression. In a nonhuman primate model of AIDS encephalitis induced by simian immunodeficiency virus (SIV) increased expression of chemokines MIP-1 $\alpha, \beta$, RANTES, MC-3 and IP-10 was observed in infected brains. Chemokines were expressed mainly in endothelial cells and perivascular monocytes/microglia (Sasseville et al, 1996). Increased expression of RANTES mRNA was detected in trigeminal ganglia taken from mice from five through 135 days after infection with herpes simplex virus type 1 (Halford et al, 1996). Histological evaluation indicated that only a very few mononuclear cells remained in the ganglion several days after infection despite the presence of HSV-1 latency-associated transcripts (Halford et al, 1996).

In brains from mice with lymphocytic choriomeningitis transcripts for chemokines, including IP10, RANTES, MCP-1, MIP-1 $\beta$ and MCP-3, were detected by day 3 postinfection. Three days later mRNA for lymphotactin, C10, MIP-2 and MIP-1 $\alpha$ was present (Asensio et al, 1999; Asensio and Campbell, 1997). Mice infected with mouse hepatitis virus (MHV) develop acute encephalomyelitis and chronic demyelinating disease. At the time of disease manifestation, mRNA for chemokines CRG2/IP-10, RANTES, MCP-1, -3, MIP-1 $\beta$ and MIP-2 was detected in brains and spinal cords. Astrocytes expressed IP-10 in this model (Lane et al, 1998). Increased IL-8 activity was observed in CSF from dogs with acute and chronic stages of canine distemper virus (CDV) infection characterized by multifocal demyelination in the central nervous system (Tipold et al, 1999). Mouse adenovirus -type 1 (MAV-1) causes fatal haemorrhagic encephalopathy associated with increased expression of IP10, MCP-1, MIP-1 $\alpha$, MIP- $1 \beta$ and RANTES in the CNS of C57BL/6 mice. IP-10 expression was detected in that disease by in situ hybridization on endothelium and CNS glia (Charles et al, 1999).

HIV-associated dementia is characterized by monocytic infiltration of the CNS. This feature suggested that chemokines can be involved in the pathomechanism of that disease. It has been shown that HIV transactivator protein Tat stimulates expression of MCP-1 by cultured astrocytes. Other chemokines were not detected in those cultures. Moreover, MCP-1 expression was detected in the brains and CSF of patients with HIV1 associated dementia (Conant et al, 1998). In brains from patients who died with HIV encephalitis chemokines MCP-1, MIP- $1 \alpha$ and RANTES were detected on macrophages (Sanders et al, 1998).

Intracisternal administration of anti-MIP-1 (including $\beta$-chemokines MIP- $1 \alpha$ and MIP- $1 \beta$ ) and anti-MIP-2 ( $\alpha$-chemokine similar to human GRO- $\alpha$ ) antibodies during induction of experimental pneumococcal meningitis delayed the onset of CSF inflammation (Saukkonen et al, 1990). In brains from mice with experimental Listeria meningoencephalitis, transcripts for MIP- $1 \alpha, \beta$ and MIP-2 were detected by Northern analysis $24 \mathrm{~h}$ after infection (Seebach et al, 1995). MIP-1 $\alpha$-positive cells were first seen in the lateral and third ventricles $12 \mathrm{~h}$ after disease induction. The vast majority of those cells were neutrophils. MIP-2 positive cells were seen later $(24-72 \mathrm{~h})$. At $72 \mathrm{~h}$ after disease induction, neutrophils and macrophages expressed both types of chemokines. Increased levels of MIP- $1 \alpha$ and MIP2 were detected by ELISA in CSF from infected animals with the peak at $48-72 \mathrm{~h}$ post infection (Seebach et al, 1995). Interpretation of these studies is complicated by the subsequent findings that MIP1 contained two distinct chemokines (MIP-1 $\alpha$ and MIP-1 $\beta$ ) and the atypical properties of murine MIP-1 $\alpha$, which can serve as a neutrophil chemoattractant.

In the CSF of patients with pyogenic meningitis elevated levels of IL-8, GRO- $\alpha$, MCP-1, MIP- $1 \alpha$ and $\beta$ were found (Spanaus et al, 1997). These patients suffered from pneumococcal, meningococcal and Haemophilus influenzae bacterial meningitis. Chemotactic activity of CSF for neutrophils was reduced by anti-IL8 and antiGRO- $\alpha$ antibodies. Similarly, mononuclear cell migration was diminished by a combination of anti-MCP-1, anti-MIP- $1 \alpha$ and anti-MIP- $1 \beta$ antibodies (Spanaus et al, 1997). In another study IL-8 level was increased in CSF from all patients with pyogenic meningitis but only in a few with aseptic meningitis. There was significant correlation between IL-8 level and CSF neutrophil counts in patients with nonpyogenic meningitis (Lopez-Cortes et al, 1995). Others observed relationship between CSF IL-8 and GRO- $\alpha$ levels and granulocyte counts in that compartment in patients with bacterial meningitis and between MCP-1 CSF concentration and mononuclear cell counts in CSF from patients with non-bacterial meningitis (Sprenger et al, 1996).

The therapeutic implications of chemokine expression in CNS infections are as protean as the agents responsible. Experiments using mice deficient in MIP- $1 \alpha$ indicated severe defects for response to viral infection in the lung, but decreased cardiac pathology after Coxsackie $B$ virus infection. Further, MIP- $1 \alpha$ knock-out mice infected with fungus Cryptococcus neoformans had decreased leukocyte recruitment and crypto- 


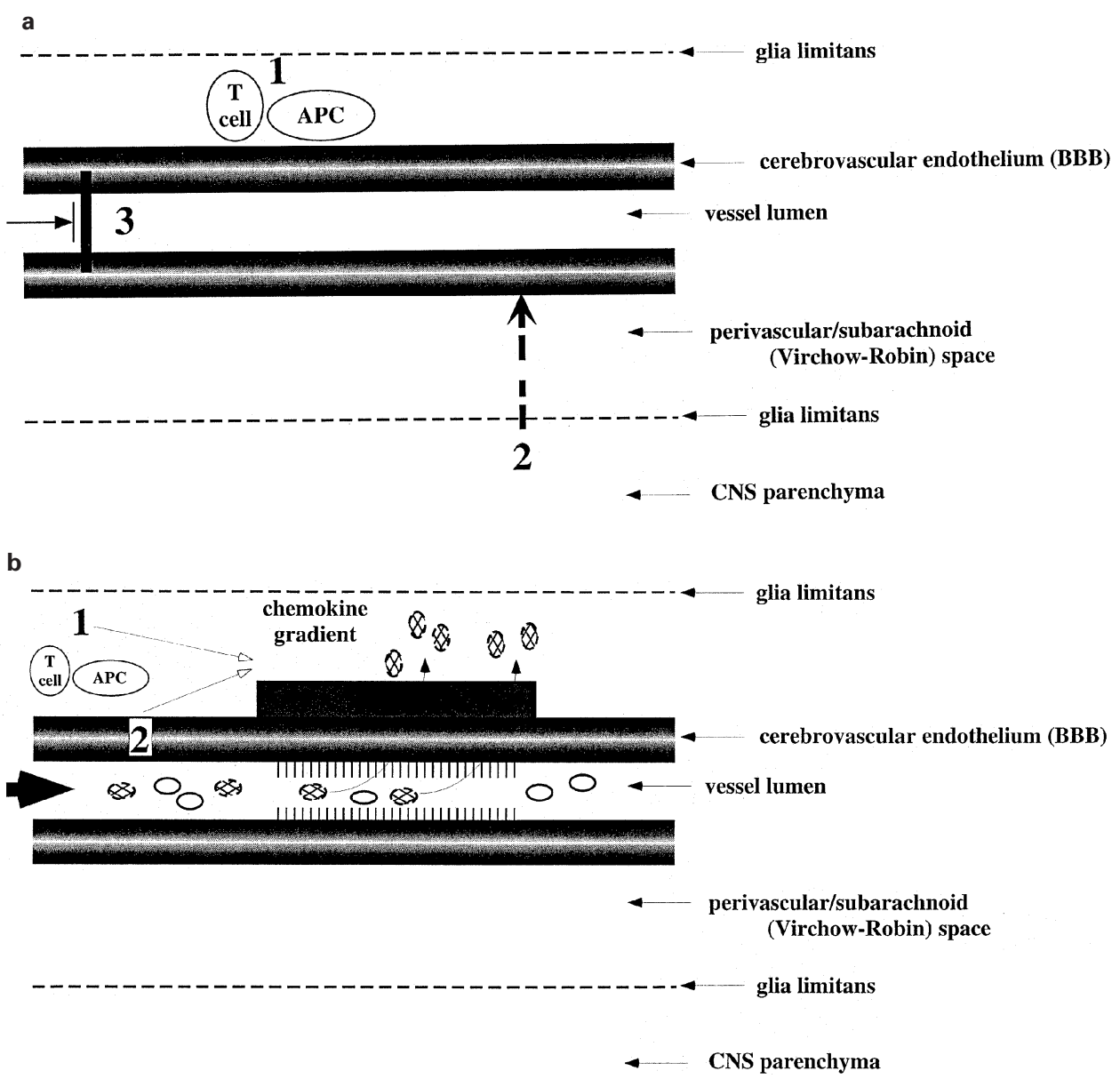

c
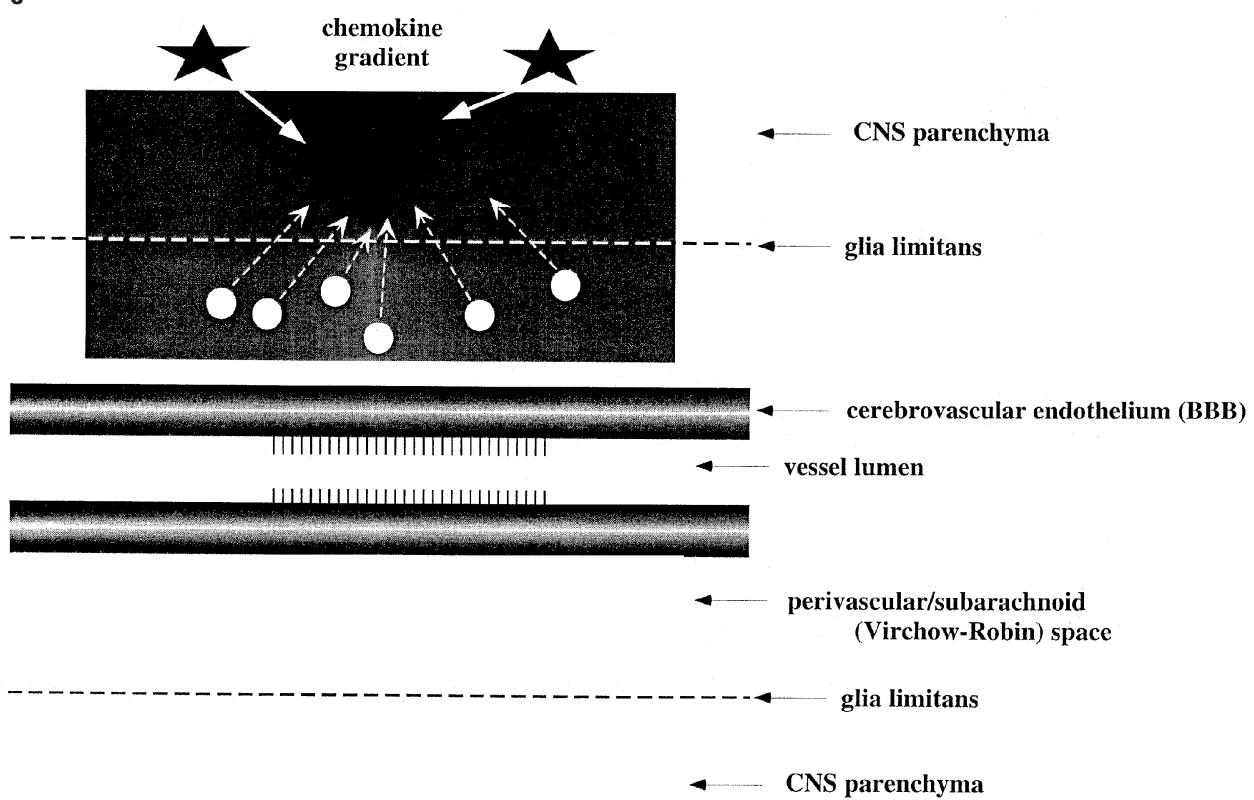

Figure 1 (a) Multiple pathways by which chemokine expression is initiated near the BBB. The cartoon shows the BBB and associated CNS structures, including the perivascular space, the glia limitans and the parenchyma. In all figures, the direction of blood flow is left-to-right. Representative pathological events are depicted. Chemokine expression can result from varied insults: (1) during immunemediated inflammation, antigen presentation and recognition by $\mathrm{T}$ cells and APCs causes chemokine production in the perivascular space, behind the BBB; (2) mechanical trauma (dashed arrow) directly impacts cellular elements of the vasculature, leading to chemokine production; (3) arterial occlusion (solid rectangle with blocked arrow) with resulting ischemic injury damages the vascular 
coccal clearance from the brain (Huffnagle and McNeil, 1999), showing the importance of chemokines in the host defense against CNS fungal infection. Cryptococcal infection stimulates IL-8 production by cultured brain glial cells and high levels of IL-8 were found in the CSF of patients with cryptococcal meningitis (Lipovsky et al, 1998). It must be recalled that expression of IL-8 by human patients with cryptococcal infection may be functionally analogous to expression of murine MIP- $1 \alpha$, since this $\beta$-chemokine can serve as a neutrophil chemoattractant. As a general comment, attempts to modulate the outcome of acute viral, bacterial or fungal infection by chemokine blockade will be attended by concern about impaired host defence. More promising is evaluation of the potential roles of chemokines and chemokine receptors in chronic viral infection, as seen in patients with HIV encephalopathy.

\section{Chemokines and the BBB}

All CNS cell types crucial for proper BBB functioning are able to express chemokines in vitro. Brain endothelial cells can produce MCP-1 and IL-8, with expression increased after stimulation with TNF- $\alpha$ or infection by bacterial parasites (Bourdoulous et al, 1995; Zach et al, 1997). Cultured normal and neoplastic astrocytes express chemokines including MCP-1 (Hayashi et al, 1995; Morita et al, 1993; Yoshimura et al, 1989), IL-8 (Morita et al, 1993; Nitta et al, 1992), IP-10 (Glabinski et al, 1999), MIP-1 $\alpha$ and $\beta$ (Miyamoto and Kim, 1999; Peterson et al, 1997) as well as RANTES (Barnes et al, 1996). TNF- $\alpha$ and IL-1 $\beta$ stimulate expression of MCP-1 and RANTES by primary human astrocytes. IFN- $\gamma$ selectively induces IP-10 expression in such cultures (Oh et al, 1999). TGF beta and IL-10 down-regulate expression of MCP-1, MIP- $1 \alpha$, MIP$1 \beta$ and RANTES by stimulated astrocytes (Guo et al, 1998). Microglia can produce IL-8, MCP-1, MIP- $1 \alpha$ and $\beta$ when cultured in vitro with inflammatory cytokines (Erlich et al, 1998; Hayashi et al, 1995; McManus et al, 1998b).
Recent studies showed that anti-MCP-1 antibody inhibited transmigration of monocytes across untreated and IL-1-stimulated cultured endothelial cells. In this system, MCP-1 stimulates transmigration of monocytes only when there is a concentration gradient across the endothelial monolayer with higher concentration in the direction of migration (Randolph and Furie, 1995). Recent studies with a model of the BBB showed that MCP-1 could induce the migration of monocytes and lymphocytes through a coculture of human endothelial cells and astrocytes. In this elegant system, stimulation with proinflammatory cytokines like TNF- $\alpha$, IFN- $\gamma$ and IL-1 $\beta$ increased monocyte transmigration, which was significantly reduced by anti-MCP-1 antibodies (Weiss et al, 1998).

Transgenic expression of chemokines behind an intact BBB, governed by an oligodendrocyte-specific promoter, showed that chemokines could direct the accumulation of specific populations of leukocytes within the brain. Specifically, MCP-1 expression under control of the MBP promoter, induced migration of monocytes/macrophages (Fuentes et $a l, 1995)$. Immunostaining detected the presence of this chemokine at the abluminal surface of cerebrospinal microvessels and infiltrating cells were localized mainly in the perivascular space (Fuentes et al, 1995). MBP-directed transgenic expression of GRO- $\alpha$ (a potent neutrophil chemoattractant) produced massive accumulation of neutrophils in perivascular, meningeal and parenchymal CNS sites. GRO- $\alpha$ immunoreactivity was detected in oligodendrocytes and colocalized with infiltrating neutrophils (Tani et al, 1996b).

Intraparenchymal chemokine expression, directed by adenoviral vectors, mediated selective recruitment of either monocytes or neutrophils depending on which chemokine was expressed (Bell et al, 1996). In these experiments, IL-8 and MIP-2 induced neutrophil accumulation, whereas MCP-1 was a potent monocyte chemoattractant. Migration of inflammatory cells correlated in these studies with BBB breakdown (Bell et al, 1996). Inflammatory cytokines IL-1 and TNF- $\alpha$ injected intracerebrally did not induce recruitment of monocytes into the brain parenchyma. Interest-

bed, causing chemokine production and BBB disruption. APC: antigen-presenting cell. (b) Simultaneous establishment of chemokine gradients and activated endothelium promote leukocyte migration across the BBB. Most insults that lead to chemokine production also upregulate adhesion molecules (vertical bars) on the luminal aspect of the endothelium. Signals that direct chemokine expression and adhesion molecule upregulation can derive either from (1) immune-mediated inflammation or 2: direct damage to the vessel wall and vascular endothelium. In either case, leukocytes (hatched circles) that express appropriate ligands for endothelial adhesion molecules (vertical bars) and for chemokine gradients in the microenvironment (shaded rectangle) will encounter conditions that favor extravasation. Leukocytes (open circles) lacking such receptors remain in the following bloodstream. (c) Leukocytes in the CNS perivascular space elicit chemokine expression by nearby glia and also respond to these chemokines. Perivascular leukocytes (white circles) are typically highly activated and produce inflammatory cytokines. These secreted products stimulate nearby astrocytes (starshaped cells) to express chemokines such as IP-10 and MCP-1, establishing a chemokine gradient (shaded rectangle) across the glia limitans. These chemokines deliver both chemoattractant and activating signals to leukocytes that express appropriate receptors. These conditions favor invasion of CNS parenchyma by leukocytes (dashed arrows) as well as persistent inflammatory microenvironment in the perivascular compartment. 
Chemokines in the BBB

AR Glabinski and RM Ransohoff ingly, these cardinal inflammatory cytokines produced neutrophil margination around vessels but not deeper migration into the brain parenchyma, showing that margination and parenchymal invasion may be regulated by distinguishable influences (Andersson et al, 1992).

\section{Chemokines, BBB and initiation of CNS inflammation}

Any cell that migrates from the blood to the CNS parenchyma has to do so in two steps. First, it transmigrates in a strictly controlled way from the vascular lumen across the BBB into the perivascular subarachnoid space (Figure 1). Second, cells within the perivascular/subarachnoid space can either remain resident there, re-enter the bloodstream (or undergo apoptosis), or can enter the CNS parenchyma. Cerebrospinal fluid (CSF) components reflect the content of the CNS extracellular fluid, consistent with the concept that the CSF-CNS barrier (glia limitans) is less restrictive than the BBB. However, the molecular mechanisms that govern infiltration of cells from the perivascular/subarachnoid space into the parenchyma remain almost completely undefined.

Chemokines may exert important functions during multiple steps of inflammatory cell extravasation (Figure 1). During the first step, chemokines immobilized on the luminal surface of the endothelium may signal to circulating leukocytes. Moreover, chemokines 'presented' by endothelial cells may stimulate expression and activation of adhesion molecules on target leukocytes and promote their attachment to the vascular endothelium (Figure 1).

When produced abluminally by CNS parenchymal cells, chemokines must be internalized and transcytosed through the endothelial cell cytoplasm to the luminal surface (Middleton et al, 1997). This concept addresses the problem that a soluble chemokine gradient cannot be maintained across an endothelium in contact with circulating blood (Tanaka et al, 1993). IL-8 immunoreactivity was shown by electron microscopy on luminal projections of endothelial cells after microinjection into rabbit skin (Middleton et al, 1997). It was suggested that heparan sulfate present on the endothelial cellsurface immobilized chemokines (Rot, 1992). This hypothesis was supported by the observation that heparitinase, an enzyme that specifically hydrolyses heparan sulfate, reduced endothelial cellassociated IL-8 immunoreactivity (Middleton et al, 1997). Additionally, heparan sulfate may also serve as counterligand for leukocyte adhesion molecules L-selectin and integrin Mac-1 (Diamond et al, 1995; Norgard-Sumnicht et al, 1993). In this view, chemokines create solid-phase rather than fluid chemotactic gradients, acting through a process termed haptotaxis. Importantly, MIP-1 $\beta$ and RANTES retain their chemotactic activities when bound to the extracellular matrix components (Gilat et al, 1994). Further, Pachter and colleagues documented high-affinity specific binding sites for beta chemokines on the abluminal surface of cerebrovascular endothelial cells (Addjelkovic et al, 1999).

Extravasation and parenchymal invasion are regulated within the CNS in a highly distinctive fashion (Figure 1). First, there are several ways that chemokine expression can be initiated in proximity to the elements of the BBB (Figure 1A). In immunemediated inflammation, leukocytes in the CNS perivascular space can promote recruitment of additional inflammatory cells from the blood by secreting inflammatory cytokines like TNF- $\alpha$ and IL$1 \beta$ that stimulate endothelial adhesion molecule expression and augment BBB leakage (Sharief et al, 1992).

Post-traumatic inflammation obviously produces direct focal impact on cellular elements of the BBB and acutely leads to chemokine production by activated endothelial elements or associated vessel-wall cells. Subsequently (days to weeks later), by unknown mechanisms, BBB function is compromised. Both neurohumoral signals and alterations in saturable transport systems have been implicated in delayed and widespread BBB decompensation after mechanical trauma.

Ischemic injury to the nervous system also directly damages the vascular bed, producing signals that elicit chemokine production. In all cases, the elaboration of chemokines, and upregulation of adhesion molecules of the immunoglobulin and selectin superfamilies on endothelial cells, appears to occur in close temporal relation. The elements are therefore established for local recruitment of leukocytes across the BBB and establishment of a perivascular inflammatory infiltrate. Further, current evidence indicates that signaling consequent to leukocyte transmigration probably leads directly to BBB disruption (Bolton et al, 1998).

Once localized in the perivascular space (Figure 1B), activated inflammatory cells may secrete chemokines like MIP- $1 \alpha$ and RANTES that can induce extravasation of additional blood-borne inflammatory cells (Glabinski et al, 1996; Miyagishi et al, 1997). When present in abundance, inflammatory cells in CNS perivascular spaces develop many of the characteristics of lymphoid organs. Among these attributes are the high-level and mutually reinforcing expression of inflammatory cytokines including IFN- $\gamma$, TNF- $\alpha$, IL-1 and IL-6. These constituents promote chemokine expression by surrounding glia, principally astrocytes. Invading leukocytes forming perivascular cuffs receive activating signals from these glial-derived chemokines and may further penetrate into the CNS parenchyma under the influence of chemokine 
gradients (Figure 1C). Parenchymal astrocytes were shown to express MCP-1, IP-10 and GRO- $\alpha$ (Ransohoff et al, 1993; Tani et al, 1996a). These sequential alterations in BBB structure and function typify

\section{References}

Ahuja S, Gao J-L, Murphy P (1994). Chemokine receptors and molecular mimickry. Immunol Today 15: 281287.

Ahuja S, Murphy $P$ (1993). Molecular piracy of mammalian interleukin-8 receptor type B by herpesvirus saimiri. J Biol Chem 268: 20691-20694.

Andersson P-B, Perry VH, Gordon S (1992). Intracerebral injection of proinflammatory cytokines or leukocyte chemotaxins induces minimal myelomonocytic cell recruitment to the parenchyma of the central nervous system. J Exp Med 176: 255-259.

Andjelkovic AV, Spencer DD and Pachter JS (1999) Visualization of chemokine binding sites on human brain microvessels. J Cell Biol 145, 403-412.

Asensio VC, Campbell IL (1997). Chemokine gene expression in the brains of mice with lymphocytic choriomeningitis. J Virol 71: 7832-7840.

Asensio V, Kincaid C, Campbell I (1999). Chemokines and the inflammatory response to viral infection in the central nervous system with a focus on lymphocytic choriomeningitis virus. J Neurovirol 5: 65-75.

Balashov KE, Rottman JB, Weiner HL, Hancock WW (1999). CCR5(+) and CXCR3(+) $\mathrm{T}$ cells are increased in multiple sclerosis and their ligands MIP-1alpha and IP-10 are expressed in demyelinating brain lesions. Proc Natl Acad Sci USA 96, 6873-6878.

Barnes D, Huston M, Holmes R, Benveniste E, Yong V, Scholz P, Perez H (1996). Induction of RANTES expression by astrocytes and astrocytoma cell lines. $J$ Neuroimmunol 71: $207-214$.

Baron JL, Madri JA, Ruddle NH, Hashim G, Janeway CA (1993). Surface expression of alpha4 integrin by CD4 $\mathrm{T}$ cells is required for their entry into brain parenchyma. J Exp Med 177: 57-68.

Bartholdi D, Schwab ME (1997). Expression of proinflammatory cytokine and chemokine mRNA upon experimental spinal cord injury in mouse: an in situ hybridization study. Eur J Neurosci, 9: 1422-1438.

Bell MD, Taub DD, Perry VH (1996). Overriding the brain's intrinsic resistance to leukocyte recruitment with intraparenchymal injections of recombinant chemokines. Neuroscience 74: 283-292.

Berman J, Guida M, Warren J, Amat J, Brosnan C (1996). Localization of monocyte chemoattractant peptide-1 expression on the central nervous system in experimental autoimmune encephalomyelitis and trauma in the rat. J Immunol 156: $3017-3023$.

Bolton SJ, Anthony DC, Perry VH (1998). Loss of the tight junction proteins occludin and zonula occludens-1 from cerebral vascular endothelium during neutrophil-induced blood-brain barrier breakdown in vivo. Neuroscience 86: $1245-1257$. chronic inflammatory processes of the CNS, such as MS. Understanding their molecular details holds significant promise for the design of novel therapeutics.

Bourdoulous S, Bensaid A, Martinez D, Sheikboudou C, Trap I, Strosberg A, Couraud P (1995). Infection of bovine brain microvessel endothelial cells with Cowdria ruminantium elicits IL-1 beta, -6 and -8 mRNA production and expression of an unusual MHC class II DQ alpha transcript. J Immunol 154: 4032-4038.

Brosnan C, Bornstein M, Bloom B (1981). The effects of macrophage depletion on the clinical and pathologic expression of experimental allergic encephalomyelitis. I Immunol 126: 614-620.

Charles P, Chen X, Horwitz M, Brosnan C (1999). Differential chemokine induction by the mouse adenovirus type- 1 in the central nervous system of susceptible and resistant strains of mice. J Neurovirol 5: $55-64$

Conant K, Garzino-Demo A, Nath A, McArthur JC, Halliday W, Power C, Gallo RC, Major EO (1998). Induction of monocyte chemoattractant protein-1 in HIV-1 Tat-stimulated astrocytes and elevation in AIDS dementia. Proc Natl Acad Sci USA, 95, 3117-3121.

Cross AH, Cannella B, Brosnan CF, Raine CS (1990). Homing to central nervous system vasculature by antigen-specific lymphocytes. I. Localization of 14Clabeled cells during acute, chronic, and relapsing experimental allergic encephalomyelitis. Lab Invest 63: $162-170$.

Diamond M, Parkos C, Quinn M, Springer T. (1995). Heparin is an adhesive ligand for the leukocyte integrin Mac-1 (CD11b/CD18). J Cell Biol 130: 14731482.

Ehrlich L, Hu S, Sheng W, Sutton R, Rockswold G, Peterson P, Chao C. (1998). Cytokine regulation of human microglial cell IL-8 production. J Immunol 160: $1944-1948$

Fuentes M, Durham S, Swerdel M, Lewin A, Barton D, Megill J, Bravo R, Lira S (1995). Controlled recruitment of monocytes/macrophages to specific organs via transgenic expression of MCPÒ1. I Immunol 155: $5769-5776$.

Gaetani P, Tartara F, Pignatti P, Tancioni F, Rodriquez Y, Baena R, De Benedetti F (1998). Cisternal CSF levels of cytokines after subarachnoid hemorrhage. Neurol Res 20: $337-342$.

Ghirnikar R, Lee Y, He T, Eng L (1996). Chemokine expression in rat stab wound brain injury. J Neurosci Res 46: $727-733$.

Ghirnikar R, Lee Y, Li J, Eng L (1998). Chemokine inhibition in rat stab wound brain injury using antisense oligodeoxynucleotides. Neurosci Lett 247: $21-24$. 
Gilat D, Hershkoviz R, Mekori Y, Vlodavsky I, Lider O (1994). Regulation of adhesion of CD4+ T lymphocytes to intact or heparinase-treated subendothelial extracellular matrix by diffusible or anchored RANTES and MIP-1 beta. J Immunol 153: 4899-4906.

Glabinski A, Krakowski M, Han Y, Owens T, Ransohoff $\mathrm{R}$ (1999). Chemokine expression in GKO mice (lacking interferon-gamma) with experimental autoimmune encephalomyelitis. J Neurovirol 5: 95-101.

Glabinski A, Tani M, Aras S, Stoler M, Tuohy V, Ransohoff R (1995a). Regulation and function of central nervous system chemokines. International $J$ Dev Neurosci 13: 153-165.

Glabinski A, Tani M, Balasingam V, Yong VW, Ransohoff RM (1996). Chemokine monocyte chemoattractant protein-1 (MCP-1) is expressed by astrocytes after mechanical injury to the brain. J Immunol 156: 4363 4368.

Glabinski A, Tani M, Strieter R, Tuohy V, Ransohoff R (1997). Synchronous synthesis of a- and b-chemokines by cells of diverse lineage in the central nervous system of mice with relapses of experimental autoimmune encephalomyelitis. Am J Pathol 150: 617630.

Glabinski A, Tani M, Tuohy VK, Tuthill R, Ransohoff RM (1995b). Central nervous system chemokine gene expression follows leukocyte entry in acute murine experimental autoimmune encephalomyelitis. Brain Behav Immun 9, 315-330.

Glabinski A, Tuohy V, Ransohoff R (1998). Expression of chemokines RANTES, MIP-1 alpha and GRO-alpha correlates with inflammation in acute experimental autoimmune encephalomyelitis. Neuroimmunomodulation 5: $166-171$.

Godiska R, Chantry D, Dietsch G, Gray P (1995). Chemokine expression in murine experimental autoimmune encephalomyelitis. $J$ Neuroimmunol 58: $167-176$.

Gourmala NG, Buttini M, Limonta S, Sauter A, Boddeke HW (1997). Differential and time-dependent expression of monocyte chemoattractant protein-1 mRNA by astrocytes and macrophages in rat brain: effects of ischemia and peripheral lipopolysaccharide administration. J Neuroimmunol 74: 35-44.

Grzybicki D, Moore S, Schelper R, Glabinski A, Ransohoff R, Murphy S (1998). Expression of monocyte chemoattractant protein (MCP-1) and nitric oxide synthase-2 following cerebral trauma. Acta Neuropathol 95: 98-103.

Guo H, Jin Y, Ishikawa M, Huang Y, van der Meide P, Link H, Xiao B (1998). Regulation of beta-chemokine mRNA expression in adult rat astrocytes by lipopolysaccharide, proinflammatory and immunoregulatory cytokines. Scand J Immunol 48: 502-508.

Halford W, Gebhardt B, Carr D (1996). Persistent cytokine expression in trigeminal ganglion latently infected with herpes simplex virus type 1. J Immunol 157: $3542-3549$.

Hausmann E, Berman N, Wang Y-Y, Meara J, Wood G, Klein R (1998). Selective chemokine mRNA expression following brain injury. Brain Res 788, 49-59.

Hayashi M, Luo Y, Laning J, Strieter RM, Dorf ME (1995). Production and function of monocyte chemoattractant protein-1 and other beta-chemokines in murine glial cells. J Neuroimmunol 60: 143-150.
Hickey WF, Hsu BL, Kimura H (1991). T-lymphocyte entry into the central nervous system. J Neurosci Res 28: $254-260$.

Huffnagle G, McNeil L (1999). Dissemination of C. neoformans to the central nervous system: role of chemokines, Th1 immunity and leukocyte recruitment. J Neurovirol 5: 76-81.

Hulkower K, Brosnan CF, Aquino DA, Cammer W, Kulshcrestha S, Guida MP, Rapoport DA, Berman JW (1993). Expression of CSF-1, c-fms and MCP-1 in the central nervous system of rats with experimental allergic encephalomyelitis. J Immunol 150: 25252533.

Hvas J, McLean C, Justesen J, Kannourakis G, Steinman L, Oksenberg J, Bernard C (1997). Perivascular T cells express the proinflammatory chemokine RANTES mRNA in multiple sclerosis lesions. Scand J Immunol 46: $195-203$.

Ivacko J, Szaflarski J, Malinak C, Flory C, Warren J, Silverstein F (1997). Hypoxic-ischemic injury induces monocyte chemoattractant protein-1 expression in neonatal rat brain. $J$ Cereb Blood Flow Metab 17: $759-770$

Jiang Y, Salafranca M, Adhikari S, Xia Y, Feng L, Sonntag M, deFiebre C, Pennell N, Streit W, Harrison $\mathrm{J}$ (1998). Chemokine receptor expression in cultured glia and rat experimental allergic encephalomyelitis. $J$ Neuroimmunol 86: $1-12$

Johansson BB (1990). The physiology of the blood-brain barrier. In: Circulating regulatory factors and neuroendocrine function. Porter JC, Jezova D (eds). Plenum Press: New York. pp. 25-39.

Karin N, Szafer F, Mitchell D, Gold D, Steinman L (1993). Selective and nonselective stages in homing of $\mathrm{T}$ lymphocytes to the central nervous system during experimental allergic encephalomyelitis. J Immunol 150: $4116-4124$

Karpus W, Ransohoff R (1998). Commentary: Chemokine regulation of experimental autoimmune encephalomyelitis: temporal and spatial expression patterns govern disease pathogenesis. J Immunol 161: 26672671.

Karpus WJ, Kennedy KJ (1997). MIP-1alpha and MCP-1 differentially regulate acute and relapsing autoimmune encephalomyelitis as well as Th1/Th2 lymphocyte differentiation. J Leukoc Biol 62: 681-687.

Karpus WJ, Lukacs NW, McRae BL, Strieter RM, Kunkel SL, Miller SD (1995). An important role for the chemokine macrophage inflammatory protein- $1 \alpha$ in the pathogenesis of the $\mathrm{T}$ cell -mediated autoimmune disease, experimental autoimmune encephalomyelitis. I Immunol 155: 5003-5010.

Kennedy K, Stieter R, Kunkel S, Lukacs N, Karpus W (1998). Acute and relapsing experimental autoimmune encephalomyelitis are regulated by differential expression of the CC chemokines macrophage inflammatory protein-1 and monocyte chemotactic protein 1. J Neuroimmunol 92: 98-108.

Kim JS, Gautam SC, Chopp M, Zaloga C, Jones ML, Ward PA, Wellch KMA (1995). Expression of monocyte chemoattractant protein-1 and macrophage inflammatory protein-1 after focal cerebral ischemia in the rat. $J$ Neuroimmunol 56: 127-134. 
Kossmann T, Stahel PF, Lenzlinger PM, Redl H, Dubs RW, Trentz O, Schlag G, Morganti-Kossmann MC (1997). Interleukin-8 released into the cerebrospinal fluid after brain injury is associated with blood-brain barrier dysfunction and nerve growth factor production. J Cereb Blood Flow Metab 17: 280-289.

Kuchroo VK, Martin CA, Greer JM, Ju S-T, Sobel RA, Dorf ME (1993). Cytokines and adhesion molecules contribute to the ability of myelin proteolipid proteinspecific $\mathrm{T}$ cell clones to mediate experimental allergic encephalomyelitis. J Immunol 151: 4371-4382.

Lane T, Asensio V, Yu N, Paoletti A, Campbell I, Buchmeier M (1998). Dynamic regulation of alphaand beta-chemokine expression in the central nervous system during mouse hepatitis virus-induced demyelinating disease. J Immunol 160: 970-978.

Lipovsky M, Gekker G, Hu S, Erhrlich L, Hoepelman A, Peterson P (1998). Cryptococcal glucuronoxylomannan induces interleukin (IL)-8 production by human microglia but inhibits neutrophil migration toward IL-8. J Infect Dis 177: 260-263.

Liu T, Young PR, McDonnell PC, White RF, Barone FC, Feuerstein GZ (1993). Cytokine-induced neutrophil chemoattractant mRNA expressed in cerebral ischemia. Neurosci Lett 164: 125-128.

Lopez-Cortes LF, Cruz-Ruiz M, Gomez-Mateos J, VicianaFernandez P, Martinez-Marcos FJ, Pachon J (1995). Interleukin-8 in cerebrospinal fluid from patients with meningitis of different etiologies: Its possible role as neutrophil chemotactic factor. J Infect Dis 172: $581-$ 584.

Luster F (1998). Chemokines-chemotactic cytokines that mediate inflammation. New Engl J Med 338: 436-445.

Male D, Pryce G, Hughes C, Lantos P (1990). Lymphocyte migration into brain modelled in vitro: Controlled by lymphocyte activation, cytokines, and antigen. Cell Immunol 127: 1-11.

Matsumoto T, Ikeda K, Mukaida N, Harada A, Matsumoto Y, Yamashita J, Matsushima K (1997). Prevention of cerebral edema and infarct in cerebral reperfusion injury by an antibody to interleukin-8. Lab Invest 77: 119-125.

McManus C, Berman J, Brett F, Staunton H, Farrell M, Brosnan C (1998a). MCP-1, MCP-2 and MCP-3 expression in multiple sclerosis lesions: an immunohistochemical and in situ hybridization study. $J$ Neuroimmunol 86: 20-29.

McManus C, Brosnan C, Berman J (1998b). Cytokine induction of MIP-1 alpha and MIP-1 beta in human fetal microglia. J Immunol 160: 1449-1455.

Mehta P, Bruccoleri A, Brown H, Harry G (1998). Increase in brain stem cytokine mRNA levels as an early response to chemical-induced myelin edema. $J$ Neuroimmunol 88: $154-164$.

Middleton J, Neil S, Wintle J, Clark-Lewis I, Moore H, Lam C, Auer M, Hub E, Rot A (1997). Transcytosis and surface presentation of IL-8 by venular endothelial cells. Cell 91: $385-395$.

Miyagishi R, Kikuchi S, Fukazawa T, Tashiro K (1995). Macrophage inflammatory protein-1a in the cerebrospinal fluid of patients with multiple sclerosis and other inflammatory neurological diseases. J Neurol Sci 129: $223-227$.
Miyagishi R, Kikuchi S, Takayama C, Inoue Y, Tashiro K. (1997). Identification of cell types producing RANTES, MIP- $1 \alpha$ and MIP- $1 \beta$ in rat experimental autoimmune encephalomyelitis by in situ hybridization. J Neuroimmunol. 77: 17-26.

Miyamoto Y, Kim S (1999). Cytokine-induced production of macrophage inflammatory protein-1 alpha (MIP-1 alpha) in cultured human astrocytes. J Neurosci Res 55: $245-251$

Morita M, Kasahara T, Mukaida N, Matsushima K, Nagashima T, Nishizawa M, Yoshida M (1993). Induction and regulation of IL-8 and MCAF production in human brain tumor cell lines and brain tumor tissues. Eur Cytokine Netw 4: 351-358.

Nitta T, Allegretta M, Okumura K, Sato K, Steinman L (1992). Neoplastic and reactive human astrocytes express interleukin-8 gene. Neurosurgical Review 15, $203-207$

Norgard-Sumnicht K, Varki N, Varki A (1993). Calciumdependent heparin-like ligands for L-selectin in nonlymphoid endothelial cells. Science 261: 480483.

Oh J, Schwiebert L, Benveniste E (1999). Cytokine regulation of CC and CXC chemokine expression by human astrocytes. I Neurovirol 5: 82-94.

Pan Y, Lloyd C, Zhou H, Dolich S, Deeds J, Gonzalo JA, Vath J, Gosselin M, Ma J, Dussault B, Woolf E, Alperin G, Culpepper J, Gutierrez-Ramos JC, Gearing D (1997). Neurotactin, a membrane-anchored chemokine upregulated in brain inflammation. Nature 387: $611-617$

Peterson PK, Hu S, Salak-Johnson J, Molitor TW, Chao CC (1997). Differential production of and migratory response to beta chemokines by human microglia and astrocytes. J Infect Dis 175: 478-481.

Randolph G, Furie M (1995). A soluble gradient of endogenous monocyte chemoattractant protein-1 promotes the transendothelial migration of monocytes in vitro. J Immunol 155: 3610-3618.

Ransohoff RM, Hamilton TA, Tani M, Stoler MH, Shick HE, Major JA, Estes ML, Thomas DM, Tuohy VK (1993). Astrocyte expression of mRNA encoding cytokines IP-10 and JE/MCP-1 in experimental autoimmune encephalomyelitis. FASEB J 7: 592-602.

Rollins BJ (1997). Chemokines. Blood 90: 909-928.

Romson J, Hook B, Kunkel S, Abrams G, Schork M, Lucchesi B (1983). Reduction of the extent of ischemic myocardial injury by neutrophil depletion in the dog. Circulation 67: 1016-1023.

Rot A (1992). Binding of neutrophil attractant/activation protein-1 (interleukin-8) to resident dermal cells. Cytokine 4: $347-352$.

Sanders V, Pittman C, White M, Wang G, Wiley C, Achim C (1998). Chemokines and receptors in HIV encephalitis. AIDS 12: 1021-1026.

Sasseville VG, Smith MM, Mackay CR, Pauley DR, Mansfield KG, Ringler DJ, Lackner AA (1996). Chemokine expression in simian immunodeficiency virus-induced AIDS encephalitis. Am J Pathol 149: $1459-1467$. 
Saukkonen K, Sande S, Cioffe C, Wolpe S, Sherry B, Cerami A, Tuomanen E (1990). The role of cytokines in the generation of inflammation and tissue damage in experimental gram-positive meningitis. J Exp Med 171: $439-448$.

Seebach J, Bartholdi D, Frei K, Spanaus KS, Ferrero E, Widmer U, Isenmann S, Strieter RM, Schwab M, Pfister H, Fontana A (1995). Experimental Listeria meningoencephalitis. Macrophage inflammatory protein-1 alpha and -2 are produced intrathecally and mediate chemotactic activity in cerebrospinal fluid of infected mice. J Immunol 155: $4367-4375$.

Selmaj K (1996). Pathophysiology of the blood-brain barrier. Springer Semin Immunopathol 18: 57-73.

Sharief MK, Ciardi M, Thompson EJ (1992). Blood-brain barrier damage in patients with bacterial meningitis: Association with tumor necrosis factor-alpha but not interleukin-1 beta. J Infect Dis 166: $350-358$.

Simpson J, Newcombe J, Cuzner M, Woodroofe M (1998). Expression of monocyte chemoattractant protein-1 and other beta-chemokines by resident glia and inflammatory cells in multiple sclerosis lesions. J Neurorimmunol 84: $238-249$.

Sørensen T, Tani M, Jensen J, Pierce V, Lucchinetti C, Folcik V, Qin S, Rottman J, Sellebjerg F, Strieter R, Frederiksen J, Ransohoff R (1999). Expression of specific chemokines and chemokine receptors in the central nervous system of multiple sclerosis patients. $J$ Clin Invest 106, 807-815.

Spanaus KS, Nadal D, Pfister HW, Seebach J, Widmer U, Frei K, Gloor S, Fontana A (1997). C-X-C and C-C chemokines are expressed in the cerebrospinal fluid in bacterial meningitis and mediate chemotactic activity on peripheral blood-derived polymorphonuclear and mononuclear cells in vitro. I Immunol 158: $1956-1964$.

Sprenger H, Rosler A, Tonn P, Braune JH, Huffmann G, Gemsa D (1996). Chemokines in the cerebrospinal fluid of patients with meningitis. Clin Immunol Immunopathol 80: 155-161.

Springer TA (1994). Traffic signals for lymphocyte recirculation and leukocyte emigration: The multistep paradigm. Cell 76: $301-314$.

Sun D, Hu X, Liu X, Whitaker JN, Walker WS (1997). Expression of chemokine genes in rat glial cells: the effect of myelin basic protein-reactive encephalitogenic T cells. J Neurosci Res 48: 192-200.

Takami S, Nishikawa H, Minami M, Nishiyori A, Sato M, Akaike A, Satoh M (1997). Induction of macrophage inflammatory protein MIP-1 alpha mRNA on glial cells after focal cerebral ischemia in the rat. Neurosci Lett 227: 173-176.

Tanaka Y, Adams D, Shaw S (1993). Proteoglycans on endothelial cells present adhesion-inducing cytokines to leukocytes. Immunol Today 14: 111-115.
Tani M, Fuentes ME, Peterson JW, Trapp BD, Durham SK, Loy JK, Bravo R, Ransohoff RM, Lira SA (1996b). Neutrophil infiltration, glial reaction and neurological disease in transgenic mice expressing the chemokine N51/KC in oligodendrocytes. J Clin Invest 98: 529539.

Tani M, Glabinski AR, Tuohy VK, Stoler MH, Estes ML, Ransohoff RM (1996a) In situ hybridization analysis of glial fibrillary acidic protein mRNA reveals evidence of biphasic astrocyte activation during acute experimental autoimmune encephalomyelitis. Am J Pathol 148: $889-896$.

Tani M, Ransohoff RM. (1994). Do cytokines mediate inflammatory cell invasion of the central nervous system parenchyma? Brain Pathol, 4: 135-143.

Tipold A, Moore P, Zurbriggen A, Burgener I, Barben G, Vandevelde $M$ (1999). Early $T$ cell response in the central nervous system in canine distemper virus infection. Acta Neuropathol 97: 45-56.

Wang X, Yue T-L, Barone FC, Feuerstein GZ (1995). Monocyte chemoattractant protein-1 messenger RNA expression in rat ischemic cortex. Stroke 26: 661666.

Weiss J, Downie S, Lyman W, Berman J (1998). Astrocyte-derived monocyte-chemoattractant protein-1 directs the transmigration of leukocytes across a model of the human blood-brain barrier. J Immunol 161: $6896-6903$

Wekerle H, Linnington C, Lassmann H, Meyermann R (1986). Cellular immune reactivity within the CNS. Trends Neurosci 9: 271-277.

Yamasaki Y, Matsuo Y, Matsuura N, Onodera H, Itoyama Y, Kogure K (1995). Transient increase of cytokineinduced neutrophil chemoattractant, a member of the interleukin-8 family, in ischemic brain areas after focal ischemia in rats. Stroke 26: $318-323$.

Yednock TA, Cannon C, Fritz LC, Sanchez-Madrid F, Steinman L, Karin N (1992). Prevention of experimental autoimmune encephalomyelitis by antibodies against alpha4beta1 integrin. Nature 356: 63-66.

Yoshimoto T, Houkin K, Tada M, Abe H (1997). Induction of cytokines, chemokines and adhesion molecule mRNA in a rat forebrain reperfusion model. Acta Neuropathol 93: 154-158.

Yoshimura T, Robinson EA, Tanaka S, Appella E, Kuratsu J, Leonard EJ (1989). Purification and amino acid analysis of two human glioma-derived monocyte chemoattractants. J Exp Med 169: 1449-1459.

Zach O, Bauer HC, Richter K, Webersinke G, Tontsch S, Bauer H (1997). Expression of a chemotactic cytokine (MCP-1) in cerebral capillary endothelial cells in vitro. Endothelium 5: 143-153. 